\title{
PHYSICAL PARAMETERS AND CHEMICAL COMPOSITION OF COMPONENTS OF THE Am-TYPE BINARY SYSTEM RR LYNCIS
}

\author{
L.S.LYUBIMKOV, T.M.RACHKOVSKAYA \\ Crimean Astrophysical Observatory, Crimea, p.Nauchny, 334413, \\ Ukraine
}

Duplicity is a very widespread phenomenon among Am-stars. For instance, Abt (1961) investigating 25 such stars found out that 22 of them are spectroscopic binaries. However this important phenomenon is ignored usually in chemical composition investigations of Am-stars. Consequently some "mean" element abundances are determined, which can noticeably differ from real abundances in atmospheres of components. Moreover false chemical anomalies can appear, as shown by the theoretical modelling of spectra of binary stars (Lyubimkov, 1989, 1992). Meanwhile accurate data on chemical composition of Am-stars must be considered as observational test for any hypothesis suggested for explanation of these objects.

Taking into account a significance of discussed problem we analyse a composite spectrum of the eclipsing binary RR Lyn (its period $P=9^{d} .945$ ), which was classified earlier as Am-star. Kondo (1976) studied its orbital elements and determined masses $M$, radii $R$ and surface gravities $g$ for both components. Moreover Kondo tried to analyse individual chemical composition of the components. As can be seen from his data, the secondary component B shows a tendency to underabundance of chemical elements relatively to the primary component $A$. At the same time there is a great scatter (about $3 \mathrm{dex}$ ) in values $\log \varepsilon_{B}-\log \varepsilon_{A}$, abundance difference between B and A. Our aim was a more accurate determination of chemical composition of the components. For solution of this task we made additional observations of RR Lyn and used more accurate technique of analysis (Lyubimkov and Samedov, 1987).

Spectroscopic observations of RR Lyn have been carried out at the Crimean Astrophysical Observatory with the $2.6 \mathrm{~m}$ telescope equipped with the CCD-camera. The usual $\mathrm{S} / \mathrm{N}$ ratio was about 100 , and dispersion was equal to $3 \AA / \mathrm{mm}$. Equivalent widths of spectral lines of both components have been measured, and these data were used in abundance analysis as well as the measurements of Kondo (1976). Observed equivalent widths were corrected in order to obtain intrinsic widths of the components $\mathrm{A}$ and $\mathrm{B}$. It is interesting that both our CCD-spectra and the data of Kondo led to compatible abundances.

The values $\log g_{A}=3.91 \pm 0.01$ and $\log g_{B}=4.07 \pm 0.08$ were adopted as surface gravities of the components A and B according to Kondo (1976). Effective 
temperatures $T_{\text {eff }}$ of the components were found by us on the basis of the following characteristics: observed equivalent widths of Balmer lines $\mathrm{H}_{\alpha}$ and $\mathrm{H}_{\gamma}$, photometric index $\left[c_{1}\right]$ and ratio of luminosities $L_{B} / L_{A}$. We must note that observations give not individual equivalent widths of Balmer lines and index $\left[c_{1}\right]$ for the components, but only common characteristics. In Fig.1 we have plotted a diagram for $T_{e f f}(\mathrm{~A})$ and $T_{\text {eff }}(\mathrm{B})$ determination. For $\mathrm{H}_{\alpha}$ and $\mathrm{H}_{\gamma}$ the average curve is drawn. For the ratio $L_{B} / L_{A}$ two cases are shown: line I corresponds to $L_{B} / L_{A}$ value of Kondo (1976), line II represents data of other authors. On the basis of this diagram we found that $T_{\text {eff }}(\mathrm{A})=8020 \pm 200 \mathrm{~K}$ and $T_{\text {eff }}(\mathrm{B})=7150 \pm 300 \mathrm{~K}$.

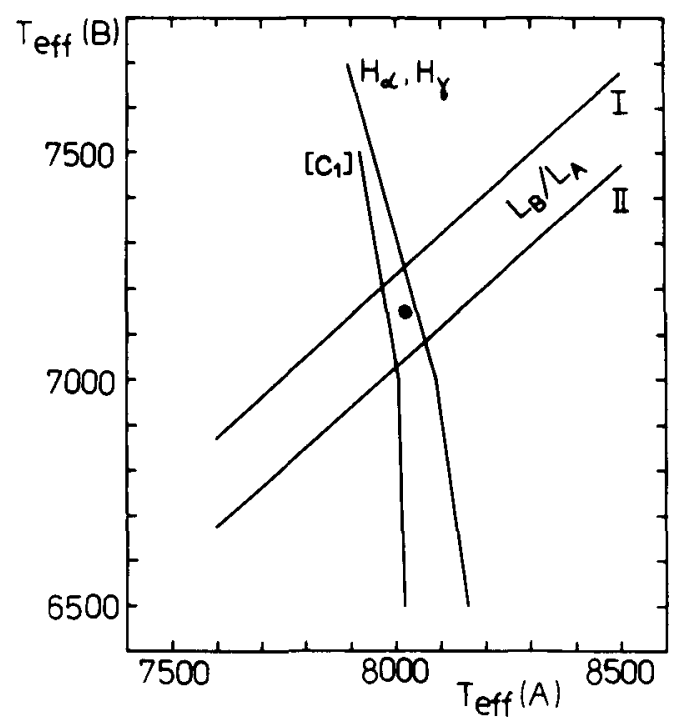

Fig. 1. Diagram for the determination of effective temperature of the components $\mathrm{A}$ and $\mathrm{B}$. Filled circle corresponds to the adopted temperatures.

A comparison of $T_{e f f}$ and $\log g$ values with evolutionary calculations allows us to evaluate the masses of components $M_{A}$ and $M_{B}$. Using evolutionary tracks of different authors we infer the values $M_{A}=1.95 \pm 0.06 M_{\odot}$ and $M_{B}=1.57 \pm 0.07 M_{\odot}$, which are in good accordance with masses $M_{A}=1.89 \pm 0.07$ $M_{\odot}$ and $M_{B}=1.50 \pm 0.05 M_{\odot}$ found by Kondo (1976) from an analysis of orbital elements. We conclude that both components correspond obviously to the main sequence evolutionary phase and are of the age $t=(1.1 \pm 0.3) * 10^{9}$ years.

Knowing parameters $T_{\text {eff }}$ and $\log g$ and also masses $M_{A}$ and $M_{B}$ we can evaluate a difference $\Delta m_{v}$ between $\mathrm{A}$ and $\mathrm{B}$ in its visual magnitudes (Lyubimkov and Samedov, 1987). We found that $\Delta m_{v}=-1.2$.

In order to determine a chemical composition of the components $A$ and 

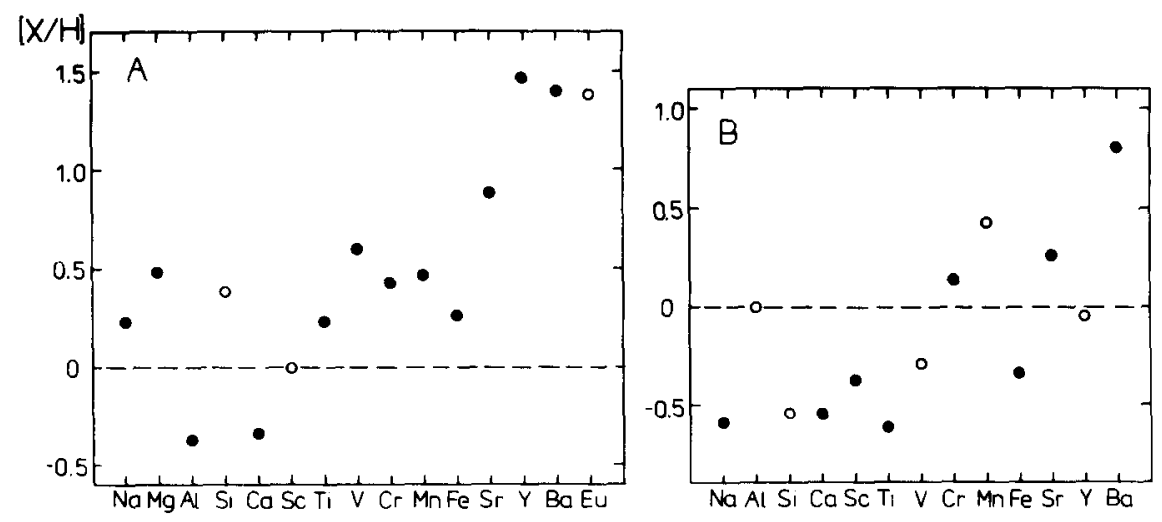

Fig. 2. Chemical composition of the components $A$ and $B$ with reference to the Sun. Open circles correspond to less confident abundances determined from one spectral line.

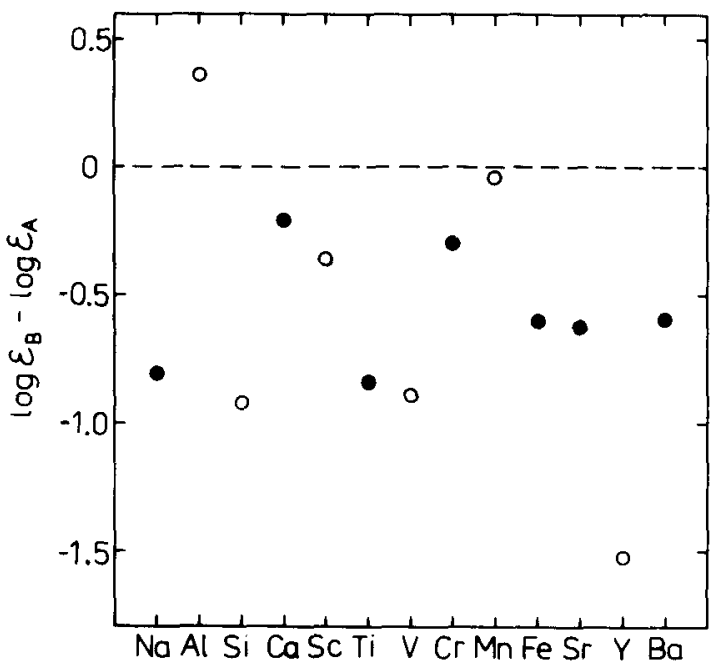

Fig. 3. Difference in chemical composition of components. 
$\mathrm{B}$, we must know its microturbulent velocities $\xi_{t}$. Using FeI lines we obtained $\xi_{t}=6.3 \pm 0.5 \mathrm{~km} / \mathrm{s}$ for the component $\mathrm{A}$. In the case of the component B this method is not successful because of insufficient number of FeI lines and considerable scatter in corresponding values of Fe abundance. Here we used the relation between $\xi_{t}$ and parameters $T_{e f f}$ and $\log g$, which has been found by Nissen (1981) for yellow dwarfs. According to this relation $\xi_{t}=2.1 \mathrm{~km} / \mathrm{s}$ for the component B.

Model atmospheres were constructed for stars A and B in accordance with its $T_{\text {eff }}$ and $\log g$ values (by interpolation of models of Kurucz, 1979). Then the chemical composition is analysed on a basis of corrected equivalent widths. In Fig. 2 determined abundances are plotted in logarithmic scale with reference to the Sun. All solar abundances with the exception of iron are taken from the compilation of Anders and Grevesse (1989). The solar iron abundance $\log \varepsilon_{\odot}(\mathrm{Fe})=7.55$ was adopted.

As can be seen from Fig.2, the component $A$ has a chemical composition, which is typical for Am-stars. Many chemical elements show overabundance, which has a tendency to increase with atomic number $\mathrm{Z}$. The greatest excess (about 1.4-1.5 dex) is found for the heaviest of considered elements - Y, Ba and Eu. For the component $B$ on the contrary most of elements is in deficiency, but in this case analogous trend with $\mathrm{Z}$ is observed, too.

So the components of the binary system RR Lyn have different chemical composition. This conclusion becomes more obvious if we calculate the abundance difference $\log \varepsilon_{B}-\log \varepsilon_{A}$. As can be seen from Fig.3, there is a systematic discrepancy in abundances between components $B$ and $A$. If uncertain points for $\mathrm{Al}$ and $\mathrm{Y}$ are excluded (its abundances $\log \varepsilon_{B}$ determined from one spectral line), we can see that all remaining values $\log \varepsilon_{B}-\log \varepsilon_{A}$ vary from 0.0 to -0.9 . On the average this difference equals -0.56 . Our analysis shows that such steady underabundance of elements in component $B$ relatively component A evidently cannot be explained by probable errors in equivalent widths or in adopted parameters $T_{\text {eff }}$ and $\log g$.

Components of the binary system have formed simultaneously and had the same primary chemical composition. We have pointed out above that at present both stars are still on the main sequence. The following questions arise: 1) what process led to the appearance of chemical peculiarities in atmospheres of stars A and B? 2) why the character of these peculiarities is different? Special interest is excited by the systematic deficiency of elements in component B relatively component A (Fig.3). As known, analogous underabundance (but relatively the Sun) was found for A-stars of $\lambda$ Boo type. One of the potential mechanisms suggested for explanation both Am and $\lambda$ Boo stars is the diffusion in the presence of mass loss. It was shown that a mass loss rate of $10^{-15} M_{\odot} / y r$ is required for appearance of Am-star, and a rate of $10^{-13} M_{\odot} / y r$ is required for appearance of $\lambda$ Boo star (Michaud, 1988).

We intend to continue the investigations of binary Am-stars to obtain more complete data on differences in chemical composition of its components. 
Detailed version of this paper will be published in Crimean Journal of Astrophysics from Radio to Gamma Rays.

\section{REFERENCES}

Abt, H.A. 1961, Ap. J. Suppl., 6, 37.

Anders, E., Grevesse, N. 1989, Geochim. Cosmochim. Acta, 53, 197.

Kondo, M. 1976, Ann. Tokyo Astron. Obs., second ser., 16, 1.

Kurucz, R.L. 1979, Ap. J. Suppl., 40, 1.

Lyubimkov, L.S. 1989, Astrofizika, 31, 519.

Lyubimkov, L.S. 1992, Izv. Krym. Astrofiz. Obs., 84, 3.

Lyubimkov, L.S., Samedov, Z.A. 1987, Izv. Krym. Astrofiz. Obs., 77, 97.

Michaud, G. 1988, in Atmospheric Diagnostics of Stellar Evolution:

Chemical Peculiarity, Mass Loss, and Explosion, ed. K.Nomoto, Springer-Verlag, Berlin, p.3.

Nissen, P.E. 1981, Astron. Astrophys., 97, 145. 